\title{
Factor de crecimiento de hepatocitos séricos como herramienta diagnóstica en pacientes con dolor torácico agudo
}

\author{
Serum hepatocyte growth factor as diagnostic tool in patients with acute chest pain
}

\author{
M. Consuelo Pintado,2*, Lara Maceda ${ }^{3}$, María Trascasa ${ }^{1}$, Aarón Blandino-Ortiz", \\ Ignacio Arribas ${ }^{5}$ y Raúl de Pablo 2,4
}

${ }^{1}$ Unidad de Cuidados Intensivos, Hospital Universitario Príncipe de Asturias; ${ }^{2}$ Departamento de Medicina y Especialidades Médicas, Facultad, Universidad de Alcalá; ${ }^{3}$ Departamento de Bioquímica, Hospital Universitario Príncipe de Asturias; ${ }^{4}$ Unidad de Cuidados Intensivos, Hospital Universitario Ramón y Cajal; ${ }^{5}$ Departamento de Bioquímica, Hospital Universitario Ramón y Cajal. Madrid, España

\section{Resumen}

Objetivo: Determinar si los niveles plasmáticos de factor de crecimiento de hepatocitos podrían ayudar a realizar el diagnóstico diferencial en pacientes con dolor torácico prolongado y elevación de la troponina cardiaca, y evaluar su valor pronóstico de mortalidad al año en estos pacientes. Método: Estudio prospectivo observacional. Se incluyeron pacientes mayores de 18 años que acudieron a urgencias con dolor torácico agudo de más de 20 minutos y elevación de la troponina cardiaca, con seguimiento al año. Resultados: Se incluyeron 303 pacientes, 103 (34\%) con infarto de miocardio y 200 (66\%) con otras enfermedades. Los niveles plasmáticos del factor de crecimiento de hepatocitos fueron superiores en el grupo sin infarto de miocardio: 329 pg/ml (rango intercuartílico [IQR]: 66-558) vs. 476 pg/ml (IQR: 264-908; p < 0.001). La mortalidad al año fue del $30.7 \%$, superior en el grupo sin infarto de miocardio (36.5\% vs. $19.4 \% ; p=0.002)$. Se encontró una fuerte asociación entre la mortalidad y los niveles elevados de factor de crecimiento de hepatocitos (650 pg/ml [344-1159] vs. $339 \mathrm{pg} / \mathrm{ml}$ [205-607]; $p$ < 0.001). En el análisis multivariado se halló que los niveles de factor de crecimiento de hepatocitos, la edad y la escala GRACE son factores independientes de mortalidad al año en estos pacientes. Conclusiones: En los pacientes con dolor torácico agudo prolongado y elevación de la troponina cardiaca, la determinación de los niveles del factor de crecimiento de hepatocitos no permite confirmar ni descartar la presencia de infarto agudo de miocardio. No obstante, podría ser un marcador pronóstico de mortalidad en estos pacientes, junto con la edad y la escala GRACE.

Palabras clave: Factor de crecimiento de hepatocitos. Síndrome coronario agudo. Infarto agudo de miocardio. Dolor torácico.

\section{Abstract}

Objective: To determine if plasma levels of hepatocyte growth factor could help in the differential diagnosis of patients with prolonged chest pain and elevated cardiac troponin; and to evaluate its prognostic value for one-year mortality in these patients. Method: A prospective observational study. Patients over the age of 18 who were seen in the emergency room for acute chest pain lasting longer than 20 minutes and elevated cardiac troponin were included, with follow up after one year. Results: We included 303 patients, 103 (34\%) with myocardial infarction and 200 (66\%) with other diseases. Plasma levels of hepatocyte

\section{Correspondencia:}

${ }^{*} \mathrm{M}$. Consuelo Pintado

E-mail: consuelopintado@yahoo.es
Disponible en internet: 07-01-2022

Rev Colomb Cardiol. 2021;28(6):564-572

www.rccardiologia.com 0120-5633 / @ 2021 Sociedad Colombiana de Cardiología y Cirugía Cardiovascular. Publicado por Permanyer. Este es un artículo open access bajo la licencia CC BY-NC-ND (http://creativecommons.org/licenses/by-nc-nd/4.0/). 
growth factor were higher in the group without myocardial infarction: 329 pg/ml (IQR: 166-558) vs. $476 \mathrm{pg} / \mathrm{ml}$ (IQR: 264-908; $p<0.001$ ). One-year mortality was $30.7 \%$, higher in the group without myocardial infarction (36.5\% vs. $19.4 \% ; p=0.002)$. We found a strong association between mortality and elevated levels of hepatocyte growth factor (650 pg/ml [344-1,159] vs. $339 \mathrm{pg} / \mathrm{ml}$ [205-607]; $p<0.001$ ). Multivariate analysis showed that levels of hepatocyte growth factor, age and the GRACE scale are independent factors for one-year mortality in these patients. Conclusions: In patients with prolonged acute chest pain and elevated cardiac troponin, hepatocyte growth factor levels do not confirm or rule out acute myocardial infarction, although they may be a prognostic marker for mortality in these patients, along with age and the GRACE scale.

Key words: Hepatocyte growth factor. Acute coronary syndrome. Acute myocardial infarction. Chest pain.

\section{Introducción}

La enfermedad coronaria es la causa principal de muerte en los países desarrollados, donde presenta, además, una elevada incidencia ${ }^{1}$. Son necesarios su diagnóstico y tratamiento precoz para disminuir su mortalidad, especialmente en los casos en que cursa como síndrome coronario agudo ${ }^{2,3}$. Se estima que menos de un $30-40 \%$ de los pacientes que acuden al servicio de urgencias con dolor torácico agudo tienen en realidad un síndrome coronario agudo ${ }^{4-6}$. Determinar qué pacientes presentan un síndrome coronario agudo frente a otras etiologías de dolor torácico agudo es un reto, en especial en aquellos que no presentan síntomas típicos o cuyo electrocardiograma (ECG) no es diagnóstico o tienen patrones anormales en ECG previos.

Aunque se han estudiado varios biomarcadores útiles para el diagnóstico y la estratificación del riesgo de los pacientes con síndrome coronario agudo (como la creatina cinasa, la mioglobina y el péptido natriurético cerebral), la determinación de las troponinas cardiacas de alta sensibilidad (hs-cTnl) es en la actualidad el biomarcador de elección para establecer el diagnóstico de infarto agudo de miocardio (IAM) ${ }^{2,3,7}$.

Aun así, se necesita otro marcador para el diagnóstico y el pronóstico del síndrome coronario agudo, por diversas razones. Los valores séricos de troponina cardiaca tienen un ascenso inicial en sangre periférica pasadas 3 a 4 horas tras del IAM (menor tiempo en caso de hs-cTnl), con un pico máximo a las 24 horas del inicio del IAM, y pueden persistir elevados hasta pasadas 2 semanas del evento isquémico; lo anterior supone un problema en determinados escenarios clínicos, como en el reinfarto precoz o en casos de elevada sospecha clínica de síndrome coronario agudo con valores de troponina normales. Por ello, se recomienda la determinación de una segunda troponina cardiaca sérica pasadas 3 horas de la primera ( 1 hora en caso de hs-CTnl) para confirmar o descartar la presencia de un síndrome coronario agudo, y de IAM en su caso; en este sentido, un segundo biomarcador podría acortar el tiempo hasta el diagnóstico ${ }^{2,7}$. Existen otras enfermedades que pueden simular un síndrome coronario agudo, con o sin alteraciones en el ECG e incluso con elevación de los niveles de troponina cardiaca sérica ${ }^{1,2,8}$. Actualmente, con la mejoría de la sensibilidad y la especificidad de los test de troponina, los falsos positivos no deberían ser un problema ${ }^{9}$.

El factor de crecimiento de hepatocitos (HGF) es producido por las células estromales y se elimina de la circulación a través del hígado. Inicialmente fue identificado como un mitógeno de los hepatocitos y luego se ha objetivado que posee también otros efectos en varios tipos celulares: regenerativos, antiapoptóticos y antifibróticos ${ }^{10}$. En los estudios clínicos se ha observado que los niveles plasmáticos de HGF presentan un ascenso precoz en los pacientes con IAM, seguido de un rápido descenso en las primeras 24 horas tras el IAM, para posteriormente mantenerse estables durante hasta 1 año del evento ${ }^{11-13}$. En otros estudios se ha observado que los niveles de HGF son un marcador de arteriosclerosis ${ }^{14}$ e IAM $^{15}$, y se ha asociado a un peor pronóstico en pacientes con síndrome coronario agudo ${ }^{11}$. Ninguno de estos estudios ha comparado los niveles de HGF con las cifras de troponina en pacientes con síndrome coronario agudo.

El objetivo principal de este estudio es determinar la utilidad clínica y diagnóstica de la determinación del HGF en pacientes con dolor torácico agudo y elevación de la troponina de alta sensibilidad para detectar los casos de síndrome coronario agudo. El objetivo secundario es determinar el valor pronóstico de mortalidad a 1 año de seguimiento de los niveles de HGF determinados al ingreso.

\section{Método}

Se trata de un estudio prospectivo y observacional realizado en un hospital universitario en Madrid, España, durante un periodo de 8 meses. Fue aprobado por el Comité de Ética y Ensayos Clínicos del Hospital Universitario Príncipe de Asturias. Los pacientes incluidos firmaron su consentimiento por escrito. Ninguno de 
los investigadores ni los pacientes incluidos en el estudio recibieron dinero ni incentivos materiales por su participación. Tampoco se recibió soporte logístico ni financiación fuera del gasto propio de los servicios del hospital implicados en el estudio.

Se incluyeron todos los pacientes que de manera consecutiva acudieron al servicio de urgencias del hospital con sospecha de síndrome coronario agudo y que dieron su consentimiento para el estudio (dolor torácico agudo y elevación de la hs-cTnl $>0.05 \mathrm{ng} / \mathrm{ml}$ ). Se excluyeron los pacientes menores de 18 años, con enfermedad en fase terminal, esperanza de vida menor de 1 año o que denegaran su consentimiento para participar en el estudio.

El diagnóstico final fue establecido por el cardiólogo responsable del enfermo, sin relación con este estudio, de acuerdo con la historia clínica, el ECG, el ecocardiograma y la determinación de troponina cardiaca según las guías internacionales del síndrome coronario agudo ${ }^{2,3,7}$.

De cada paciente se recolectaron características demográficas, factores de riesgo de síndrome coronario agudo, antecedente de síndrome coronario agudo, enfermedades concomitantes, tratamiento actual, escalas de riesgo GRACE (Global Registry of Acute Coronary Events) ${ }^{16}$ y TIMI (Thrombolysis In Myocardial Infarction $)^{17}$, y mortalidad hospitalaria y al año. El seguimiento se realizó mediante revisión de la historia clínica o llamada telefónica al año, o ambos.

Tras su inclusión en el estudio, se identificaron y recuperaron las muestras de sangre tomadas en urgencias en el momento de la valoración inicial para su análisis. Se obtuvieron muestras de plasma tras centrifugación de las muestras de sangre (tomadas en heparina-litio BD Vacutanier ${ }^{\circledR}$ ) durante 7 minutos a 3500 r.p.m., que fueron divididas en alícuotas de $0.5 \mathrm{ml}$ y almacenadas congeladas a $-40^{\circ} \mathrm{C}$ para la posterior determinación del HGF. La cuantificación de la troponina de alta sensibilidad se realizó en muestras de plasma en el momento de la valoración en urgencias mediante el analizador ADVIA Centaur XP ${ }^{\circledR}$ TnI-Ultra ${ }^{\circledR}$ (Siemens), realizándose determinaciones seriadas posteriores cada 6 horas hasta alcanzar el pico máximo. La concentración mínima medible con el uso de este test, que presenta un coeficiente de variación $<10 \%$, es de $30 \mathrm{ng} / \mathrm{l}$. El HGF se midió en las muestras de plasma descongeladas tomadas en el momento de su evacuación en urgencias mediante ensayo inmunoabsorbente ligado a enzimas $\left(\mathrm{Abcam}^{\circledR}\right)$. El nivel mínimo de detección fue de $3 \mathrm{pg} / \mathrm{ml}$, con un coeficiente de variación del $10 \%$. La determinación de la creatina cinasa se realizó también en el momento de la evaluación en urgencias y procesada en el analizador ADVIA Chemistry 1800 (Siemens).

El objetivo primario del estudio fue establecer la relación entre los niveles séricos de HGF y el diagnóstico de síndrome coronario agudo en pacientes no seleccionados que presentaban dolor torácico agudo y niveles elevados de hs-cTnl.

Los objetivos secundarios fueron determinar la relación entre los niveles de HGF y la mortalidad hospitalaria y al año, así como los factores relacionados con la mortalidad a 1 año en estos pacientes.

Para el análisis estadístico se determinó qué variables cuantitativas presentaban una distribución normal mediante el test de Kolmogórov-Smirnov. Aquellas variables cuantitativas que presentaron una distribución normal se expresan como media \pm desviación estándar y se compararon con el test $t$ de Student; en caso contrario, se describen como mediana y rango intercuartílico (IQR), y se compararon mediante el test de Mann-Whitney. Las variables cualitativas se expresan como porcentajes y se compararon con el test de chi al cuadrado.

Se estableció como nivel de significación estadística una $p<0.05$, usando contraste bilateral, y los resultados se expresan con un intervalo de confianza del 95\% (IC95\%).

La eficacia diagnóstica del HGF se ha evaluado mediante su sensibilidad, especificidad, índice de probabilidad y valores predictivos positivo y negativo.

Se construyó un modelo de regresión logística para el análisis multivariado con el fin de identificar los factores asociados con la mortalidad al año de seguimiento. Primero se realizó un análisis univariado de las principales variables recogidas para evaluar de manera individual su significación estadística frente a la mortalidad al año; aquellas que tuvieron significación estadística fueron incluidas en un modelo inicial para un análisis de regresión logística múltiple paso a paso hacia adelante. Los resultados se expresan como razón de disparidad con su IC95\%.

El análisis estadístico fue llevado a cabo con el programa estadístico SPSS 15.0 (SPSS Inc., Chicago, Illinois).

\section{Resultados}

Se incluyeron en total 303 pacientes en el estudio. La edad media fue de 76 años (64-85 años) y la mayoría eran varones (56.1\%). El factor de riesgo para síndrome coronario agudo más frecuente fue la presencia de hipertensión arterial (75.2\%), seguido del tabaquismo $(47.9 \%)$ y la dislipidemia (45.9\%) (Tabla 1$)$. 
Tabla 1. Características basales de los pacientes incluidos en el estudio

\begin{tabular}{|c|c|c|c|c|}
\hline & $\begin{array}{l}\text { Todos los } \\
\text { pacientes } \\
(\mathrm{n}=303)\end{array}$ & $\begin{array}{l}\text { Síndrome } \\
\text { coronario agudo } \\
(\mathbf{n}=103)\end{array}$ & $\begin{array}{l}\text { Sin síndrome } \\
\text { coronario agudo } \\
(\mathrm{n}=200)\end{array}$ & $\mathbf{p}$ \\
\hline Edad, años ${ }^{\dagger}$ & $76.0(64.0-85.0)$ & $71.0(60.0-83.0)$ & $79.0(65.0-86.0)$ & $0,008^{*}$ \\
\hline Sexo, masculino & $170(56.1 \%)$ & $65(63.1 \%)$ & $105(52.5 \%)$ & 78 \\
\hline $\begin{array}{l}\text { Factores de riesgo para síndrome coronario agudo } \\
\text { Hipertensión arterial } \\
\text { Diabetes mellitus } \\
\text { Dislipidemia } \\
\text { Antecedentes familiares de enfermedad cardiovascular } \\
\text { Tabaquismo } \\
\text { Antecedentes de enfermedad arterial coronaria }\end{array}$ & $\begin{array}{c}228(75.2 \%) \\
112(37.0 \%) \\
139(45.9 \%) \\
2(0.7 \%) \\
145(47.9 \%) \\
83(27.4 \%)\end{array}$ & $\begin{array}{c}79(76.7 \%) \\
47(45.6 \%) \\
62(60.2 \%) \\
2(1.9 \%) \\
64(62.1 \%) \\
36(35.0 \%)\end{array}$ & $\begin{array}{c}149(74.5 \%) \\
65(32.5 \%) \\
77(38.5 \%) \\
0(0.0 \%) \\
81(40.5 \%) \\
47(23.5 \%)\end{array}$ & $\begin{array}{c}0.674 \\
0.025^{*} \\
<0.001^{*} \\
0.115 \\
<0.001^{*} \\
0.034^{*}\end{array}$ \\
\hline $\begin{array}{l}\text { Comorbilidad } \\
\text { Hiperuricemia } \\
\text { Enfermedad renal } \\
\text { Enfermedad pulmonar } \\
\text { Enfermedad hepática } \\
\text { Miocardiopatía } \\
\text { Valvulopatía }\end{array}$ & $\begin{array}{c}22(7.3 \%) \\
65(21.5 \%) \\
84(27.7 \%) \\
3(1.0 \%) \\
32(10.6 \%) \\
25(8.3 \%)\end{array}$ & $\begin{array}{c}5(4.9 \%) \\
14(13.6 \%) \\
22(21.4 \%) \\
1(1.0 \%) \\
2(1.9 \%) \\
4(3.9 \%)\end{array}$ & $\begin{array}{c}17(8.5 \%) \\
51(25.5 \%) \\
62(31.0 \%) \\
2(1.0 \%) \\
30(15.0 \%) \\
21(10.5 \%)\end{array}$ & $\begin{array}{c}0.247 \\
0.017^{*} \\
0.076 \\
0.900 \\
<0.001^{*} \\
0.047^{*}\end{array}$ \\
\hline $\begin{array}{l}\text { Valoración del riesgo isquémico } \\
\text { Escala de riesgo GRACE } \\
\text { Escala de riesgo TIMI }\end{array}$ & $\begin{array}{c}130.0(100.0-153.0) \\
3.0(2.0-4.0)\end{array}$ & $\begin{array}{c}115.0(93.5-143.0) \\
3.0(2.7-4.0)\end{array}$ & $\begin{array}{c}137.5(107.0-156.7) \\
2.0(2.0-3.0)\end{array}$ & $\begin{array}{c}0.003^{*} \\
<0.001^{*}\end{array}$ \\
\hline
\end{tabular}

Resultados expresados como número (porcentaje), excepto los marcados con ${ }^{\dagger}$, que están expresados como mediana (rango intercuartílico). ${ }^{*} \mathrm{p}<0.05$. GRACE: Global Registry of Acute Coronary Events; TIMI: Thrombolysis In Myocardial Infarction.

Tabla 2. Diagnóstico definitivo de los pacientes sin síndrome coronario agudo

\begin{tabular}{|l|l|}
\hline Sepsis grave y shock séptico & $73(36.5 \%)$ \\
\hline \begin{tabular}{l} 
Insuficiencia cardiaca grave \\
\hline Taquiarritmia/bradiarritmia
\end{tabular} & $45(22.5 \%)$ \\
\hline Embolia pulmonar & $29(14.5 \%)$ \\
\hline $\begin{array}{l}\text { Contusión cardiaca o procedimientos cardiacos } \\
\text { (cardioversión, marcapasos) }\end{array}$ & $14(7.0 \%)$ \\
\hline Miocarditis/miopericarditis & $11(5.5 \%)$ \\
\hline \begin{tabular}{l} 
Evento neurológico agudo \\
\hline $\begin{array}{l}\text { Comorbilidad cardiaca en pacientes con } \\
\text { insuficiencia renal crónica }\end{array}$
\end{tabular} & $9(4.5 \%)$ \\
\hline $\begin{array}{l}\text { Enfermedad infiltrativa cardiaca } \\
\text { Emergencia hipertensiva }\end{array}$ & $6(3.0 \%)$ \\
\hline $\begin{array}{l}\text { Enfermedad estructural cardiaca (miocardiopatía } \\
\text { hipertrófica, estenosis aórtica) }\end{array}$ & $2(2.0 \%)$ \\
\hline Resultados expresados como número (porcentaje). & $4(2.0 \%)$ \\
\hline
\end{tabular}

\section{Niveles de HGF en los pacientes con síndrome coronario agudo confirmado frente a otras causas}

De los 303 pacientes incluidos en el estudio, 103 (34\%) tenían síndrome coronario agudo confirmado y $200(66 \%)$ tenían otras causas de dolor torácico y elevación de la hs-cTnl.

A la inclusión, los pacientes con otras causas de dolor torácico (no síndrome coronario agudo) eran mayores (79.0 años [65.0-86.0] vs. 71.0 años [60.0-83.0]; $p=0.008$ ], con mayor incidencia de enfermedad renal crónica (25.5\% de los pacientes sin síndrome coronario agudo vs. $13.6 \%$ en el grupo de síndrome coronario agudo; $p=0.017$ ), miocardiopatía ( $15.0 \%$ en pacientes sin síndrome coronario agudo vs. $1.9 \% ; p<0.001)$ e historia de enfermedad valvular cardiaca ( $10.5 \%$ en pacientes $\sin$ síndrome coronario agudo vs. 3.9\%; $p=0.047$ ). Los pacientes con síndrome coronario agudo confirmado tenían mayor incidencia de arteriosclerosis coronaria ( $35.0 \%$ vs. $23.5 \%$; $p=0.034)$, diabetes mellitus $(45.6 \%$ vs. $32.5 \%$; $p=0.025)$, dislipidemia $(60.2 \%$ vs. $38.5 \% ; p<0.001)$ y tabaquismo (62.1\% vs. $40.5 \%$; $p<0.001$ ) (Tabla 1).

De los 103 pacientes con diagnóstico de síndrome coronario agudo, $28(27.2 \%)$ fueron finalmente diagnosticados de IAM con elevación persistente del segmento ST y 75 (72.8\%) de síndrome coronario agudo sin elevación persistente del segmento ST (74 con IAM sin elevación del ST y uno con miocardiopatía de Takotsubo). Entre los 200 pacientes que no fueron diagnosticados con síndrome coronario agudo, el diagnóstico más frecuente fue daño miocárdico en el contexto de sepsis y shock séptico (36.5\%) e insuficiencia cardiaca grave (22.5\%) (Tabla 2). 
Tabla 3. Valores de HGF, hs-cTnl y creatina quinasa

\begin{tabular}{|l|c|c|c|c|}
\hline & $\begin{array}{c}\text { Todos los pacientes } \\
(\mathbf{n}=303)\end{array}$ & $\begin{array}{c}\text { Síndrome coronario agudo } \\
(\mathrm{n}=103)\end{array}$ & $\begin{array}{c}\text { Sin síndrome coronario agudo } \\
(\mathbf{n}=200)\end{array}$ & \begin{tabular}{c} 
p \\
\hline HGF $(\mathrm{pg} / \mathrm{ml})$
\end{tabular} \\
\hline $421(226-798)$ & $329(166-558)$ & $476(264-908)$ & $<0.001$ \\
\hline hs-cTnl a la inclusión (ng/ml) & $0.2(0.1-0.7)$ & $0.6(0.1-4.4)$ & $0.2(0.1-0.4)$ & $<0.001$ \\
\hline Creatina cinasa (UI/l) a la inclusión & $110(70-191)$ & $139(91-335)$ & $90(62-171)$ & $<0.001$ \\
\hline
\end{tabular}

Resultados expresados como mediana (rango intercuartílico).

HGF: factor de crecimiento de hepatocitos; hs-cTnl: troponina I cardiaca de alta sensibilidad

Tabla 4. Sensibilidad y especificidad de los valores de HGF

\begin{tabular}{|c|c|c|c|c|}
\hline \multicolumn{5}{|c|}{ HGF para diagnóstico de síndrome coronario agudo } \\
\hline & Síndrome coronario agudo & Sin síndrome coronario agudo & & \\
\hline \multirow[t]{3}{*}{$\begin{array}{l}\mathrm{HGF}(\mathrm{pg} / \mathrm{ml}) \\
>421 \\
<421\end{array}$} & $\begin{array}{l}41 \\
62\end{array}$ & $\begin{array}{c}110 \\
90\end{array}$ & $\begin{array}{l}151 \\
152\end{array}$ & $\begin{array}{l}\text { VPP: } 27.2 \% \\
\text { VPN: } 59.2 \%\end{array}$ \\
\hline & 103 & 200 & & \\
\hline & Sensibilidad: $39.8 \%$ & Especificidad: $45.0 \%$ & & \\
\hline \multicolumn{5}{|c|}{ HGF como factor pronóstico de mortalidad al año } \\
\hline & Fallecidos & Supervivientes & & \\
\hline \multirow[t]{3}{*}{$\begin{array}{l}\mathrm{HGF}(\mathrm{pg} / \mathrm{ml}) \\
>421 \\
<421\end{array}$} & $\begin{array}{l}56 \\
31\end{array}$ & $\begin{array}{c}95 \\
121\end{array}$ & $\begin{array}{l}151 \\
152\end{array}$ & $\begin{array}{l}\text { VPP: } 37.1 \% \\
\text { VPN: } 79.6 \%\end{array}$ \\
\hline & 87 & 216 & & \\
\hline & Sensibilidad: $64.4 \%$ & Especificidad: $56.0 \%$ & & \\
\hline
\end{tabular}

HGF: factor de crecimiento de hepatocitos; VPN: valor predictivo negativo; VPP: valor predictivo positivo.

En la tabla 3 se muestran los niveles de HGF, hs-cTnl y creatina cinasa observados. Cabe señalar que los niveles de HGF que se obtuvieron fueron más bajos en los pacientes con síndrome coronario agudo que en los que no lo presentaban: $329 \mathrm{pg} / \mathrm{ml}$ (IQR: 166-558) vs. $476 \mathrm{pg} / \mathrm{ml}$ (IQR: 264-908; $\mathrm{p}<0.001)$.

Para un punto de corte de $421 \mathrm{pg} / \mathrm{ml}$ (mediana del nivel de HGF medido en todos los pacientes incluidos en el estudio), la sensibilidad del HGF para diagnosticar síndrome coronario agudo fue del $39.8 \%$, con una especificidad del $45.0 \%$, un valor predictivo positivo del $27.2 \%$, un valor predictivo negativo del $59.2 \%$ y un índice de probabilidad del 2.4 (IC95\%: 1.4-3.9; $p=0.001$ ) (Tabla 4).

De los 303 pacientes incluidos en el estudio, $268(88.4 \%)$ fueron ingresados en el hospital (165 [82.5\%] pacientes sin síndrome coronario agudo y todos los pacientes con síndrome coronario agudo; $\mathrm{p}<0.001$ ).
La mortalidad global hospitalaria fue del $19.8 \%$. Se encontró mayor mortalidad hospitalaria en los pacientes $\sin$ síndrome coronario agudo $(25.5 \%$ vs. $10.7 \% ; p=0.003$ ). Los niveles de HGF observados al ingreso fueron mayores en los pacientes que fallecieron en el hospital (741 pg/ml [363-1751] en los pacientes fallecidos vs. $382 \mathrm{pg} / \mathrm{ml}$ [217-726]; $p<0.001)$.

Al año de seguimiento, la mortalidad global fue del $30.7 \%$, también superior en los pacientes no diagnosticados de síndrome coronario agudo ( $36.5 \%$ vs. $19.4 \%$; $p=0.002)$, y con niveles de HGF observados al ingreso mayores en los pacientes que habían fallecido al año de seguimiento $(650 \mathrm{pg} / \mathrm{ml}$ [344-1759] en los pacientes fallecidos vs. $339 \mathrm{pg} / \mathrm{ml}$ [205-606]; $p<0.001]$. En un análisis multivariado de la mortalidad al año se encontró que una puntuación en la escala GRACE > 118, la edad y los valores de HGF > $421.24 \mathrm{pg} / \mathrm{ml}$ (mediana 
Tabla 5. Factores relacionados con la mortalidad a 1 año de seguimiento

\begin{tabular}{|c|c|c|}
\hline & OR (IC95\%) & $\mathbf{p}$ \\
\hline $\begin{array}{l}\text { Análisis univariado } \\
\text { Sexo masculino } \\
\text { Edad } \\
\text { Antecedente de enfermedad } \\
\text { arterial coronaria } \\
\text { HGF }>421.24 \mathrm{pg} / \mathrm{ml} \\
\text { Puntuación escala GRACE > } 118 \\
\text { Puntuación escala TIMI > } 4 \\
\text { Infarto de miocardio } \\
\text { Diabetes mellitus } \\
\text { Hipertensión sistémica } \\
\text { Tabaquismo }\end{array}$ & $\begin{array}{c}0.929(0.568-1.518) \\
1.076(1.050-1.102) \\
1.411(0.826-2.408) \\
2.379(1.435-3.939) \\
7.425(3.822-14.423) \\
0.793(0.355-1.772) \\
\\
0.419(0.238-0.739) \\
1.114(0.673-1.842) \\
2.080(1.109-3.899) \\
0.262(0.124-0.554)\end{array}$ & $\begin{array}{l}0.767 \\
0.000 \\
0.207 \\
0.001 \\
0.000 \\
0.572 \\
\\
0.003 \\
0.675 \\
0.022 \\
0.000\end{array}$ \\
\hline $\begin{array}{l}\text { Análisis multivariado } \\
\text { Puntuación escala } \\
\text { GRACE }>118 \\
\text { Edad } \\
\text { HGF }>421.24 \mathrm{pg} / \mathrm{ml}\end{array}$ & $\begin{array}{l}2.592(1.066-6.300) \\
1.049(1.016-1.083) \\
2.003(1.155-3.472)\end{array}$ & $\begin{array}{l}0.003 \\
0.013\end{array}$ \\
\hline
\end{tabular}

HGF: Factor de crecimiento de hepatocitos; OR: razón de disparidad (odds ratio).

de los resultados obtenidos) son factores independientes de mortalidad al año de seguimiento (Tabla 5).

\section{Discusión}

En este estudio se observa que la determinación del HGF sérico en el momento de la valoración en urgencias en pacientes no seleccionados con dolor torácico prolongado y elevación de la hs-cTnl no permite confirmar ni descartar la presencia de síndrome coronario agudo. Nuestros resultados sugieren que la determinación del HGF podría ser un biomarcador para predecir la mortalidad al año de seguimiento en esta población de pacientes, junto con la edad y una puntuación elevada en la escala de riesgo de GRACE $^{16}$, independientemente de si se confirma el diagnóstico o no de síndrome coronario agudo. Por consiguiente, la determinación conjunta del HGF y la hs-cTnl podría ser útil para estratificar mejor el riesgo y el manejo de estos pacientes.

Varios estudios han demostrado que los niveles de HGF son superiores en los pacientes con síndrome coronario agudo frente a los encontrados en voluntarios sanos ${ }^{18-21}$, aunque sin diferencias estadísticamente significativas entre los pacientes con IAM 0 angina inestable ${ }^{18}$. Estos niveles alcanzan un pico máximo al ingreso por IAM, permanecen estables durante 7 días y descienden de forma gradual posteriormente ${ }^{12,20}$. Solo un estudio ha encontrado una correlación entre los niveles precoces de HGF y la extensión del IAM ${ }^{20}$.
Sin embargo, cuando se comparan los niveles séricos de HGF en los pacientes con enfermedad cardiaca isquémica o sin ella, los resultados con contradictorios. Algunos estudios ${ }^{15,21,22}$ muestran que los niveles de HGF son significativamente superiores en los pacientes con IAM 0 angina inestable frente a los de aquellos con angina de esfuerzo estable ${ }^{21}$, astenia neurocirculatoria ${ }^{22} u$ otras enfermedades cardiacas ${ }^{15}$, lo que sugiere que puede ser una herramienta útil para realizar el diagnóstico diferencial en estos pacientes. Sin embargo, otros autores, como es nuestro caso, han encontrado que, aunque los niveles de HGF están elevados en los pacientes con síndrome coronario agudo, también están elevados en los pacientes con dolor torácico debido a otras causas no relacionadas con isquemia arterial coronaria, como insuficiencia cardiaca de origen no isquémico ${ }^{23}$, disección aórtica aguda 0 tromboembolia pulmonar $^{24}$, sin diferencias en los niveles séricos de HGF entre los diferentes subgrupos.

Algunos autores postulan que la elevación de los niveles de HGF encontrados en los pacientes con isquemia miocárdica se debe a la presencia de una respuesta inflamatoria que aparece tras la isquemia aguda. Otros investigadores que apoyan esta teoría han demostrado la existencia de una correlación entre los niveles séricos de HGF y los niveles de proteína C reactiva en los pacientes con IAM O angina inestable $^{18,20}$. Sin embargo, Soeki, et al. ${ }^{19}$ encontraron que, en los pacientes con IAM, la elevación de los niveles de HGF se debe mayoritariamente a fuentes no cardiacas, ya que la concentración de HGF que hallaron en el seno coronario no difiere de la encontrada en muestras periféricas. La respuesta inflamatoria puede ser mayor en otras patologías que también inducen dolor torácico diferentes del síndrome coronario agudo, unido al hecho de que el HGF es un marcador de daño en el tejido vascular humano ${ }^{10,25,26}$, lo que puede explicar los elevados niveles séricos de HGF encontrados en otras enfermedades, como ocurre en la hipertensión arterial sistemica ${ }^{27}$, la enfermedad arterial periférica sintomática ${ }^{28}$, la fibrilación auricular ${ }^{29}$ y la falla renal aguda $^{30}$.

Nuestros resultados sugieren que, en los pacientes con dolor torácico agudo con elevación de la hs-cTnl, los niveles plasmáticos de HGF $>421 \mathrm{pg} / \mathrm{ml}$, la edad y una puntuación en la escala GRACE $^{16}>118$ pueden identificar a aquellos con mayor riesgo de haber fallecido al año de seguimiento. Entre los pacientes con síndrome coronario agudo, los niveles elevados de HGF se asocian a un peor pronóstico. Nuestros 
hallazgos son similares a los de otros estudios que han demostrado la existencia de una relación directa entre la elevación de los niveles de HGF y la presencia de mayor riesgo de eventos cardiovasculares adversos serios durante el seguimiento ${ }^{11,13,31}$. También se ha asociado la elevación de los niveles séricos de HGF con la mortalidad en pacientes con falla cardiaca avanzada $^{23}$. En cambio, Heeschen, et al. ${ }^{32}$ encontraron que los niveles elevados de HGF en pacientes con angina inestable refractaria se asociaban con menor mortalidad y menor tasa de IAM a los 6 meses, aunque en su estudio los pacientes con niveles mayores de HGF tenían, con mayor frecuencia, circulación coronaria colateral y llenado retrógrado parcial o completo en la coronariografía.

Los niveles séricos elevados de HGF se han asociado a un peor pronóstico en otros escenarios clínicos; por ejemplo, el desarrollo de lesiones arteriales coronarias en pacientes con enfermedad de Kawasaki ${ }^{33}$, el rechazo del injerto en el trasplante cardiaco ${ }^{34}$, la menor supervivencia en determinados tipos de cáncer ${ }^{35-37}$, el aumento de la mortalidad en la insuficiencia cardiaca avanzada $^{23}$ y la menor probabilidad de recuperación en pacientes con hemiparesia tras un ictus ${ }^{38}$. Se ha informado de que los pacientes con algunos tipos de cáncer (carcinoma pulmonar de célula pequeña, cáncer colorrectal metastásico, cáncer de ovario) que presentan niveles elevados de HGF presentan menor surpervivencia ${ }^{35-37}$. Un estudio ha observado que los niveles séricos de HGF y de factor de crecimiento placentario son factores predictores independientes de mortalidad a 10 años por todas las causas, por enfermedad cardiovascular y por cáncer en la población general ${ }^{39}$. También se ha reportado que los niveles séricos de HGF son mayores en los pacientes en hemodiálisis crónica, con carcinoma hepatocelular, en estadios avanzados de cirrosis hepática y con hepatitis crónica ${ }^{40-42}$. Se desconoce el mecanismo fisiopatológico subyacente a estos hallazgos, pero se cree que está relacionado con el daño vascular asociado en estas patologías. En estudios con animales se ha sugerido la presencia de un mecanismo endocrino relacionado con el HGF que interviene en la reparación tisular o la regeneración de los tejidos dañados ${ }^{25,26}$. Adicionalmente, podría estar relacionado con la síntesis de HGF por las células lisas musculares y los fibroblastos en los tejidos vasculares dañados por la respuesta inflamatoria generada ${ }^{43}$. Otro mecanismo posible es la activación aberrante del Met a través del HGF. El Met es un receptor transmembrana de la tirosina cinasa que es sobreexpresado en muchos tumores sólidos y que se asocia con un aumento de la movilidad, la migración, la invasión y la angiogénesis en diversos modelos tumorales ${ }^{44,45}$.

Nuestros resultados confirman la utilidad de la escala de riesgo GRACE $^{16}$, pues en este estudio se correlaciona con un peor pronóstico, no solo en pacientes con síndrome coronario agudo, sino también en aquellos con dolor torácico agudo y elevación de la hs-cTnl de origen no isquémico coronario. Como sabemos, la escala GRACE $^{16}$ estratifica el riesgo de mortalidad a los 6 meses en pacientes con síndrome coronario agudo basándose en un estudio prospectivo y multicéntrico que realizó un registro global de pacientes con síndrome coronario agudo ${ }^{16,17}$. Se ha estudiado el valor pronóstico de la escala GRACE $^{16}$ en pacientes con dolor torácico agudo en servicios de urgencias. Chen, et al. ${ }^{46}$ compararon el valor pronóstico de cuatro escalas diferentes y observaron una puntuación en la escala GRACE ${ }^{16} \geq 160$, que predice un resultado seguro a los 6 meses de seguimiento (especificidad del $91.9 \%$, sensibilidad del $64.1 \%$ y área bajo la curva [AUC] 0.843). Lyon, et al..$^{47}$ demostraron que tanto la escala GRACE ${ }^{16}$ como la TIMI $^{17}$ son efectivas para estratificar el riesgo en los pacientes con dolor torácico en los servicios de urgencias, con un AUC de 0.80 para la escala GRACE y de 0.78 para la TIMI ${ }^{17}$. No hay datos sobre el valor pronóstico de la escala $\mathrm{GRACE}^{16}$ en pacientes sin dolor torácico no secundario a síndrome coronario agudo. Es interesante que en nuestro estudio encontramos que la puntuación en la escala GRACE $^{16}$ fue más alta en los pacientes sin síndrome coronario agudo, y se asociaba a una mayor mortalidad al año de seguimiento en el análisis univariado en los pacientes con dolor torácico agudo y elevación de la hs-cTnl. Esto no solo puede deberse a que esta escala recoge la edad, la presencia de IAM previo, la insuficiencia cardiaca, los cambios en el segmento ST y la elevación de las enzimas cardiacas, como la escala TIMI ${ }^{17}$, sino también al tratamiento recibido, la presencia de falla renal aguda y la gravedad de la enfermedad basada en la exploración clínica. En nuestro estudio, cerca del $60 \%$ de los pacientes sin síndrome coronario agudo tenían sepsis, shock séptico o insuficiencia cardiaca grave, lo que puede explicar la relación encontrada entre la puntuación en la escala GRACE $^{16}$ y el pobre pronóstico.

Este estudio presenta varias limitaciones. Primero, las muestras para el estudio del HGF fueron obtenidas a la llegada a urgencias, con un tiempo de evolución variable desde el inicio del dolor (incluso a veces indeterminado debido a la imprecisión de la clínica 
referida por el paciente); así pues, los cambios rápidos iniciales de los niveles séricos observados en los pacientes con IAM pueden afectar a los resultados obtenidos. A esto se suma que solo se determinó al ingreso, sin seriación de las determinaciones. No se evaluó la respuesta inflamatoria, por lo que no se puede correlacionar con los niveles de HGF, y tampoco permite explicar por qué los pacientes que fallecen durante su estancia hospitalaria presentan niveles séricos superiores de HGF. No se aplicaron escalas de gravedad de la enfermedad, como la escala APACHE II8 o la SOFA $^{49}$, que podrían explicar la mortalidad observada en nuestro estudio. Como marcador pronóstico que es, no se ha realizado un estudio de costos amplio y adecuado que permita evaluar su eficacia en términos de costo-efectividad, el cual sería interesante realizar de cara a evaluar su utilidad en la práctica clínica. Actualmente, el precio del kit comercial en Europa es de 552 euros para 96 test.

Por otro lado, la fuerza de este estudio se basa en que se muestran datos obtenidos en el mundo real, en condiciones reales de práctica clínica, como refleja la gran tasa de pacientes valorados en urgencias por dolor torácico agudo que presentan elevación de la hs-cTnl, sin deberse a un síndrome coronario agudo, como describen otros autores ${ }^{1,2,4-6,8}$.

En conclusión, en pacientes con sospecha de IAM (dolor torácico prolongado y elevación de la hs-cTnl), la determinación de los niveles séricos de HGF no permite confirmar ni descartar la presencia de un IAM. A pesar de ello, nuestros resultados sugieren que podría ser un buen biomarcador para predecir la mortalidad hospitalaria y al año de seguimiento en estos pacientes, junto con la edad y la escala GRACE ${ }^{16}$.

\section{Financiamiento}

Este estudio no ha recibido financiamiento alguno.

\section{Conflicto de intereses}

Todos los autores declaran que no tienen ningún conflicto de intereses.

\section{Responsabilidades éticas}

Protección de personas y animales. Los autores declaran que para esta investigación no se han realizado experimentos en seres humanos ni en animales.
Confidencialidad de los datos. Los autores declaran que han seguido los protocolos de su centro de trabajo sobre la publicación de datos de pacientes.

Derecho a la privacidad y consentimiento informado. Los autores declaran que en este artículo no aparecen datos de pacientes.

\section{Bibliografía}

1. Go AS, Mozaffarian D, Roger VL, Benjamin EJ, Berry JD, Blaha MJ, et al. Executive summary: heart disease and stroke statistics - 2014 update: a report from the American Heart Association. Circulation. 2014;129:399-410.

2. Roffi M, Patrono C, Collet JP, Mueller C, Valgimigli M, Andreotti F, et al. 2015 ESC Guidelines for the management of acute coronary syndromes in patients presenting without persistent ST-segment elevation. Task Force for the Management of Acute Coronary Syndromes in Patients Presenting without Persistent ST-Segment Elevation of the European Society of Cardiology (ESC). G Ital Cardiol (Rome). 2016;17:831-72.

3. Task Force on the management of ST-segment elevation acute myocardial infarction of the European Society of Cardiology (ESC), Steg PG, James SK, Atar D, Badano LP, Blömstrom-Lundqvist C, Borger MA, et al. ESC Guidelines for the management of acute myocardial infarction in patients presenting with ST-segment elevation. Eur Heart J. 2012;33:2569-619.

4. Cullen L, Mueller C, Parsonage WA, Wildi K, Greenslade JH, Twerenbold R, et al. Validation of high-sensitivity troponin I in a 2-hour diagnostic strategy to assess 30-day outcomes in emergency department patients with possible acute coronary syndrome. J Am Coll Cardiol. 2013;62:1242-9.

5. Möckel M, Searle J, Hamm C, Slagman A, Blankenberg S, Huber K, et al. Early discharge using single cardiac troponin and copeptin testing in patients with suspected acute coronary syndrome (ACS): a randomized, controlled clinical process study. Eur Heart J. 2015;36:369-76.

6. Pascual Figal DA, Noguera Velasco JA, Rodenas Checa J, Murcia Alemán T, Martínez Cadenas J, Ferrándiz Gomis R, et al. Chest pain in clinical practice: impact of routine troponin determination on clinical manifestations and care. Rev Esp Cardiol. 2003:56:43-8.

7. Thygesen K, Alpert JS, Jaffe AS, Chaitman BR, Bax JJ, Morrow DA, et al. Fourth universal definition of myocardial infarction (2018). Circulation. 2018;138:e618-51.

8. Wildi K, Twerenbold R, Mueller C. How acute changes in cardiac troponin concentrations help to handle the challenges posed by troponin elevations in non-ACS-patients. Clin Biochem. 2015;48:218-22.

9. Chacko S, Haseeb S, Glover BM, Wallbridge D, Harper A. The role of biomarkers in the diagnosis and risk stratification of acute coronary syndrome. Future Sci OA. 2018:4:FSO251.

10. Nakamura T, Mizuno $S$. The discovery of hepatocyte growth factor (HGF) and its significance for cell biology, life sciences and clinical medicine. Proc Jpn Acad Ser B Phys Biol Sci. 2010;86:588-610.

11. Konopka A, Janas J, Piotrowski W, Stepinska J. Hepatocyte growth factor - a new marker for prognosis in acute coronary syndrome. Growth Factors. 2010;28:75-81.

12. Jiménez-Navarro MF, González FJ, Caballero-Borrego J, Marchal JA Rodríguez-Losada N, Carrillo E, et al. Coronary disease extension determines mobilization of endothelial progenitor cells and cytokines after a first myocardial infarction with ST elevation. Rev Esp Cardiol. 2011;64:1123-9.

13. Lamblin N, Bauters A, Fertin M, de Groote P, Pinet F, Bauters C. Circulating levels of hepatocyte growth factor and left ventricular remodelling after acute myocardial infarction (from the REVE-2 study). Eur J Heart Fail. 2011;13:1314-22

14. Nishimura M, Ushiyama M, Nanbu A, Ohtsuka K, Takahashi H, Yoshimura M. Serum hepatocyte growth factor as a possible indicator of arteriosclerosis. J Hypertens. 1997;15:1137-42.

15. Matsumori A, Furukawa Y, Hashimoto T, Ono K, Shioi T, Okada M, et al. Increased circulating hepatocyte growth factor in the early stage of acute myocardial infarction. Biochem Biophys Res Commun. 1996;221:391-5.

16. Fox KA, Dabbous OH, Goldberg RJ, Pieper KS, Eagle KA, Van de Werf $F$, et al. Prediction of risk of death and myocardial infarction in the six months after presentation with acute coronary syndrome: prospective multinational observational study (GRACE). BMJ. 2006;333:1091.

17. Antman EM, Cohen M, Bernink PJ, McCabe CH, Horacek T, Papuchis G, et al. The TIMI risk score for unstable angina/non-ST elevation MI: a method for prognostication and therapeutic decision making. JAMA. 2000;284:835-42.

18. Shimada $Y$, Yoshiyama $M$, Jissho $S$, Kamimori $K$, Nakamura $Y$, lida $H$, et al. Hepatocyte growth factor production may be related to the inflammatory response in patients with acute myocardial infarction. Circ $\mathrm{J}$. 2002;66:253-6. 
Rev Colomb Cardiol. 2021;28(6)

19. Soeki T, Tamura Y, Shinohara H, Sakabe K, Onose Y, Fukuda N. Serum hepatocyte growth factor predicts ventricular remodeling following myocardial infarction. Circ J. 2002;66:1003-7.

20. Zhu Y, Hojo Y, Ikeda U, Shimada K. Production of hepatocyte growth factor during acute myocardial infarction. Heart. 2000;83:450-5.

21. Sato T, Yoshinouchi T, Sakamoto T, Fujieda H, Murao S, Sato H, et al. Hepatocyte growth factor (HGF): a new biochemical marker for acute myocardial infarction. Heart Vessels. 1997:12:241-6.

22. Suzuki H, Murakami M, Shoji M, Iso Y, Kondo T, Shibata M, et al. Hepatocyte growth factor and vascular endothelial growth factor in ischaemic heart disease. Coron Artery Dis. 2003:14:301-7.

23. Rychli K, Richter B, Hohensinner PJ, Kariem Mahdy A, Neuhold S, Zorn G, et al. Hepatocyte growth factor is a strong predictor of mortality in patients with advanced heart failure. Heart 2011:97:1158-63.

24. Hata N, Matsumori A, Yokoyama S, Ohba T, Shinada T, Yoshida H, et al. Hepatocyte growth factor and cardiovascular thrombosis in patients admitted to the intensive care unit. Circ J. 2004;68:645-9.

25. Higuchi $O$, Nakamura T. Identification and change in the receptor for hepatocyte growth factor in rat liver after partial hepatectomy or induced hepatitis. Biochem Biophys Res Commun. 1991;176:599-607.

26. Yanagita $K$, Nagaike $M$, Ishibashi $H$, Niho $Y$, Matsumoto $K$, Nakamura $T$. Lung may have an endocrine function producing hepatocyte growth factor in response to injury of distal organs. Biochem Biophys Res Commun. 1992;182:802-9.

27. Hayashi $Y$, Saitoh S, Takagi S, Tuchihashi K, Miura T, Shimamoto K Hepatocyte growth factor and 24-hour ambulatory blood pressure monitoring. Hypertens Res. 2002;25:655-60.

28. Lin JW, Sheu WH, Lee WJ, Chen YT, Liu TJ, Ting CT, et al. Circulating hepatocyte growth factor level but not basic fibroblast growth factor level is elevated in angiography-proven symptomatic peripheral artery disease. Angiology. 2007;58:420-8

29. Katoh $\mathrm{H}$, Shimada $\mathrm{T}$, Inoue $\mathrm{S}$, Takahashi $\mathrm{N}$, Shimizu $\mathrm{H}$, Ohta $\mathrm{Y}$, et al. Reduced high serum hepatocyte growth factor levels after successful cardioversion in patients with atrial fibrillation. Clin Exp Pharmacol Physiol. 2004;31:145-51.

30. Libetta C, Rampino T, Esposito C, Fornoni A, Semeraro L, Dal Canton A. Stimulation of hepatocyte growth factor in human acute renal failure. Nephron. 1998;80:41-5

31. Konopka A, Janas J, Stepinska J. Hepatocyte growth factor concentration during the first day of acute coronary syndrome. Arch Med Sci. 2012;8:389-91.

32. Heeschen C, Dimmeler S, Hamm CW, Boersma E, Zeiher AM, Simoons ML; CAPTURE (c7E3 Anti-Platelet Therapy in Unstable REfractory angina) Investigators. Prognostic significance of angiogenic growth factor serum levels in patients with acute coronary syndromes. Circulation. 2003:107:524-30.

33. Ohno $\mathrm{T}$, Yuge $\mathrm{T}$, Kariyazono $\mathrm{H}$, Igarashi $\mathrm{H}$, Joh-o $\mathrm{K}$, Kinugawa $\mathrm{N}$, et al. Serum hepatocyte growth factor combined with vascular endothelial growth factor as a predictive indicator for the occurrence of coronary artery lesions in Kawasaki disease. Eur J Pediatr. 2002;161:105-11.

34. Zwirska-Korczala K, Zakliczynski M, Berdowska A. Diagnostic validity of hepatocyte growth factor as marker for rejection in the follow-up of patients after heart transplantation. J Heart Lung Transplant. 2005;24:411-5.
35. Aune G, Lian AM, Tingulstad S, Torp SH, Forsmo S, Reseland JE, et al. Increased circulating hepatocyte growth factor (HGF): a marker of epithelial ovarian cancer and an indicator of poor prognosis. Gynecol Oncol. 2011;121:402-6.

36. Cañadas I, Taus A, González I, Villanueva X, Gimeno J, Pijuan L, et al High circulating hepatocyte growth factor levels associate with epithelial to mesenchymal transition and poor outcome in small cell lung cancer patients. Oncotarget. 2014:5:5246-56.

37. Takahashi N, Yamada Y, Furuta K, Honma Y, Iwasa S, Takashima A, et al. Serum levels of hepatocyte growth factor and epiregulin are associated with the prognosis on anti-EGFR antibody treatment in KRAS wild-type metastatic colorectal cancer. Br J Cancer. 2014;110:2716-27.

38. Okazaki H, Beppu H, Mizutani K, Okamoto S, Sonoda S. Changes in serum growth factors in stroke rehabilitation patients and their relation to hemiparesis improvement. J Stroke Cerebrovasc Dis. 2014;23:1703-8.

39. Santalahti K, Havulinna A, Maksimow M, Zeller T, Blankenberg S, Vehtari $A$, et al. Plasma levels of hepatocyte growth factor and placental growth factor predict mortality in a general population: a prospective cohort study. J Intern Med. 2017;282:340-52.

40. Libetta C, Esposito P, Martinelli C, Grosjean F, Gregorini M, Rampino T, et al. Hepatocyte growth factor (HGF) and hemodialysis: physiopathology and clinical implications. Clin Exp Nephrol. 2016;20:371-8.

41. Bilezikci B, Haberal AN, Demirhan B. Hepatocyte growth factor in patients with three different stages of chronic liver disease including hepatocellular carcinoma, cirrhosis and chronic hepatitis: an immunohistochemical study. Can J Gastroenterol. 2001;15:159-65.

42. Prystupa A, Kicinski P, SakJ, Boguszewska-Czubara A, Toruń-Jurkowska A, Załuska W. Proinflammatory cytokines (IL-1 alpha, IL-6) and hepatocyte growth factor in patients with alcoholic liver cirrhosis. Gastroenterol Res Pract. 2015;2015:532-615.

43. Nakamura Y, Morishita R, Higaki J, Kida I, Aoki M, Moriguchi A, et al. Expression of local hepatocyte growth factor system in vascular tissues. Biochem Biophys Res Commun. 1995;215:483-8.

44. Trusolino L, Bertotti A, Comoglio PM. MET signalling: principles and functions in development, organ regeneration and cancer. Nat Rev Mol Cell Biol. 2010;11:834-48.

45. Natan S, Tsarfaty G, Horev J, Haklai R, Kloog Y, Tsarfaty I. Interplay Between HGF/SF-Met-Ras signaling, tumor metabolism and blood flow as a potential target for breast cancer therapy. Oncoscience. 2014;1:30-8.

46. Chen XH, Jiang HL, Li YM, Chan CPY, Mo JR, Tian CW, et al. Prognostic values of 4 risk scores in Chinese patients with chest pain: prospective 2-centre cohort study. Medicine (Baltimore). 2016;95:e4778.

47. Lyon R, Morris AC, Caesar D, Gray S, Gray A. Chest pain presenting to the emergency department - to stratify risk with GRACE or TIMI? Resuscitation. 2007:74:90-3.

48. Knaus WA, Draper EA, Wagner DP, Zimmerman JE. APACHE II: a severity of disease classification system. Crit Care Med. 1985;13:818-29.

49. Vincent JL, de Mendonca A, Cantraine F, Moreno R, Takala J, Suter PM, et al. Use of the SOFA score to assess the incidence of organ dysfunction/ failure in intensive care units: results of a multicenter, prospective study. Working group on "sepsis-related problems" of the European Society of Intensive Care Medicine. Crit Care Med. 1998:26:1793-800. 